\title{
How to Manage the COVID-19 Diffusion in the Angiography Suite: Experiences and Results of an Italian Interventional Radiology Unit
}

\author{
Marcello Andrea Tipaldi ${ }^{\text {a b* }}{ }^{*}$, Elena Lucertini ${ }^{a}$, Gianluigi Orgera ${ }^{\text {a }}$, Aleksejs Zolovkins ${ }^{\text {a }}$, \\ Florindo Lauirno a , Edoardo Ronconi ${ }^{\mathrm{a}}$, Andrea Pisano a , Patrizia Carmela La Salandra a , \\ Andrea Laghi ${ }^{\text {a }}$, Michele Rossi ${ }^{\text {a }}$ \\ a Department of Surgical and Medical Sciences and Translational Medicine, School of Medicine and Psychology, "Sapienza" - \\ University of Rome, Rome, Italy. \\ ${ }^{b}$ Consultant Vascular and Interventional Radiologist, Department of Radiology, Sant'Andrea University Hospital La Sapienza, Rome, Italy.
}

Received 18 July 2020; Accepted 07 August 2020

\begin{abstract}
Introduction: The management of the diffusion of Coronavirus disease 2019 (COVID-19) pandemic represents a massive problem for healthcare systems worldwide and Interventional Radiology (IR) is a fundamental hospital unit which must continue to provide its service. The aim of this article is to summarize the preventive measures taken in our IR unit and to report the results of these measures over a 7 weeks period. Material and Methods: Between the $25^{\text {th }}$ of February, when we started to apply the recommended containing measures, and the 6th of April 2020, when all the IR staff started to undergo nasopharyngeal and oropharyngeal swabs screening, a total of 25 healthcare operators worked at our IR unit. Operators who, during this period, also worked in other hospital units such as diagnostic emergency department or other healthcare facilities, were excluded. Nasopharyngeal and oropharyngeal swabs screening and blood samples for specific SARS-CoV-2 IgG-IgM were retrospectively evaluated. Results: The overall procedures number decreased by a rate of $33 \%$ and twenty-three (16\%) were performed in confirmed or strongly suspected COVID-19 patients. Two procedures were performed in non-suspected ones, who revealed positive in the following hospitalization days. Seventeen operators were included in the study. Only one of them resulted positive at the swabs, with an estimated infection rate in our IR unit of $6 \%$. Specific SARS-CoV-2 IgG-IgM resulted negative in all the operators included. Conclusion: Our experience demonstrates that applying adequate measures to limit SARS-CoV-2 infection spread can efficiently reduce the viral transmission among IR healthcare workers.
\end{abstract}

Keywords: Coronavirus Disease 2019; COVID-19; SARS-CoV-2; Angiographic Suite; Interventional Radiology; Coronavirus Disease; COVID-19 Pandemic; Case Management.

\section{Introduction}

The severe acute respiratory syndrome Coronavirus 2 (SARS-CoV-2) is a highly contagious virus with a very efficient human-to-human transmission, via respiratory secretions and contaminated surfaces [1]. The management of the diffusion of the Coronavirus disease 2019 (COVID-19) pandemic represents a massive problem for healthcare systems worldwide.

SARS-CoV-2 infection can cause severe respiratory symptoms which require intensive care unit (ICU) in critical cases $(2,1 \%)$ and whose case fatality rate (CFR) in Italy is up to $18 \%$ [2, 3]. Although interventional radiology (IR) is not directly involved in the treatment of COVID-19 related symptoms, it must continue to provide its service within

\footnotetext{
* Corresponding author: tipaldi.andrea@gmail.com

dol) http://dx.doi.org/10.28991/SciMedJ-2020-02-SI-1

$>$ This is an open access article under the CC-BY license (https://creativecommons.org/licenses/by/4.0/).

(C) Authors retain all copyrights.
} 
the hospital, paying attention to avoid or, at least, reduce the contagion diffusion both among patients and the team. Despite the workload is expected to decrease in a COVID Hub Hospital, IR service must be preserved and guaranteed.

Essential procedures such as life-threatening or organ-saving treatments and elective oncologic procedures, that cannot be reasonably postponed [4], must be performed. Because of these reasons, it is mandatory to apply strict and efficient measures to prevent and control the spread of COVID-19.

Based on World Health Organization (WHO) guidelines and other center's experiences [4-10], the most important principle to prevent nosocomial transmission of SARS-CoV-2 is the segregation of patients in space and time. In particular, patients with suspected or confirmed COVID-19 infection should take a dedicated route separated from the "clean" one to reach the angiographic suite. In addition, or when it is not possible to separate the route, a temporal segregation of patients is recommended. IR procedures on patients with confirmed or suspected COVID-19 should be performed after those on non-infected patients, guaranteeing an accurate cleaning and disinfection of the operating room at the end of each procedure. Negative pressure room with high efficiency particulate air filtration may be the ideal environment to avoid the viral diffusion [5]. Furthermore, a reduction of healthcare staff to the bare minimum during procedures and a segregation of staff members into independent small teams is recommended, if possible [4]. Adequate personal protective equipment (PPE) must be provided to all patients and to the staff members. PPE for operators performing a procedure on a suspected or confirmed infected patient must include masks (FFP2 or FFP3), gloves, gowns, eye protection and shoe covers [4, 5]. Access area to the room, where the personnel wears PPE, should be separated from the exit "undress" area, were the PPE can be properly disposed, before moving back to the clean area. Despite these measures, transmission to healthcare workers has proven worryingly high [11]. In Italy, healthcare operators contagion represents almost $12 \%$ of the total number of infected [2]. The aim of this article is to summarize the preventive measures taken in our IR unit and to report the results of these measures over a 7 weeks period.

\section{Materials and Methods}

This is a single center retrospective study. Due to the retrospective nature of the study, informed consent was waived by the ethics committee. All methods or experimental protocols were approved by the local Institutional review board.

\subsection{Measures Adopted Against COVID-19 Spread}

On March 2020, our hospital has become a COVID-19-Hub. This process included the closure of several wards which have been moved to other "non-COVID" hospitals and the establishment of designated units entirely dedicated to COVID-19 patients with different levels of intensive care.

Emergencies progressively became mostly dedicated to patients with COVID-19 related symptoms or to disparate symptoms in patients with confirmed or highly suspected COVID-19. Nevertheless, the Hospital was still active in providing assistance for occasional non-COVID emergencies as well as for medical or surgical oncologic patients although reducing the procedures to the bare minimum.

Due to the rapid spread or SARS-CoV-2 epidemic in Italy and the never interrupted activity of our hospital, on 25/02/2020 our IR unit started to apply the most feasible and efficient measures to contain the viral diffusion, according to the Hospital internal guidelines which were based on the WHO recommendations and to other center's experiences $[4,9]$ :

- Patients hospital access: at access to the hospital service, all patients undergo a specific interview to evaluate the infection risk degree, the possible presence of COVID-19 related symptoms and nasopharyngeal and oropharyngeal swabs with RT-PCR analysis are performed.

- PPE: both infected and non-infected patients must wear surgical mask and gloves; staff members must wear surgical mask and gloves during each patient's interview, in addition to the sterile equipment normally used during interventions. For procedures on confirmed or suspected COVID-19 patients, all operators must additionally use FFP2/FFP3 masks, double sterile gloves, gowns, goggles or face shield and shoe covers. All this equipment must be properly worn, carefully removed in a specific dedicated room and correctly disposed, following validated guidelines [10] (Figure 1).

- Reduced workload: we assessed both the infection risk and the urgency of each procedure. Only life-threatening or organ-saving treatments and oncologic procedures that could not be reasonably postponed, were maintained. Furthermore, we keep on guaranteeing all the necessary IR procedures on hospitalized patients.

- All the elective procedures and the ambulatory services have been postponed or delegated to other nonCOVID-Hub healthcare facilities. 


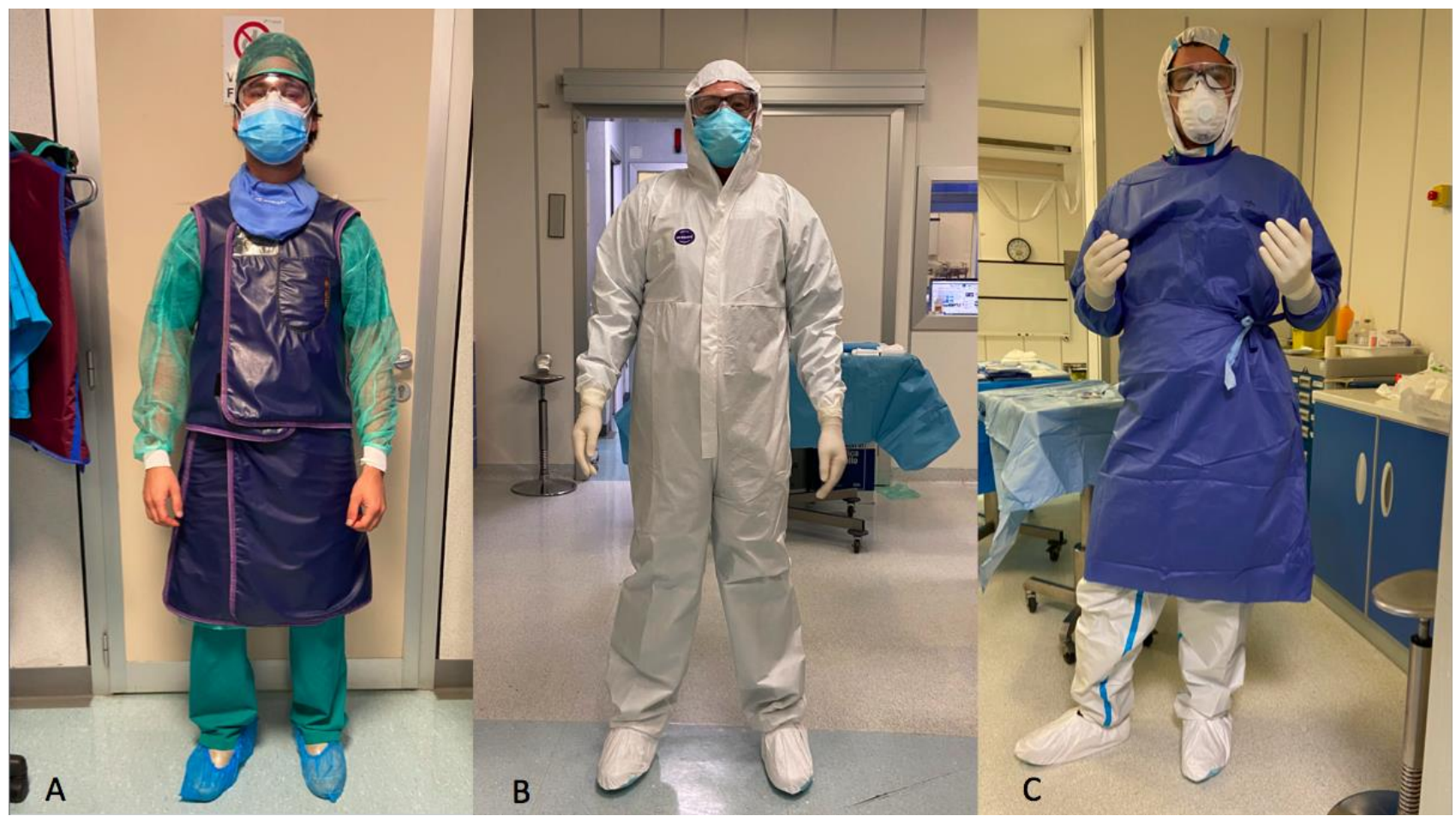

Figure 1. A-C Wearing protocol for confirmed or suspected COVID-19 patients. First step: operators wear lead gown, cap, eye protection, FFP2 or FFP3 mask, surgical mask in case of valved FFP2/3 mask and shoe covers (A); Second step: operators wear repellent coverall suite or gown and non-sterile gloves (B); Third step: operators wear sterile gown and gloves and start procedure $(\mathrm{C})$.

- Reduced number of operators and social distancing: the working team has been reduced to the bare minimum (one interventional radiologist, one resident, one radiographer and one nurse; an additional radiologist is always available in case of necessity) and each operator must wear a surgical mask during the entire working shift and is solicited to carefully wash hands frequently. When possible, a minimum distance of 1 meter has to be maintained between all healthcare operators.

O Other specialists, students or any kind of healthcare operator, not directly involved in the procedure are not allowed to attend the area.

- Time segregation of patients: due to the architectural characteristic of our IR area, it was not possible to create a specific route for COVID-19 patients totally separated from the "clean" route (Figure 2). Therefore, we decided to segregate patients in time, performing the interventions on patients with confirmed or suspected COVID-19 after all the non-infected ones, compatibly with the degree of urgency.

- Disinfection of the rooms: after procedures on COVID-19 confirmed or suspected patients, the suite, the removing PPE area and the COVID-route are deeply cleaned and disinfected. Exposed surfaces are cleaned with $70 \%$ ethanol or chlorhexidine-ethanol wipes and floors are cleaned with disinfectant, keeping them unavailable for 60-90 minutes. Differently, when more procedures on infected patients are performed, they are scheduled to be executed consecutively, without the necessity of a specific disinfection between them. After all the procedures on non-COVID-19 patients, the rooms are cleaned with virus-neutralizing products (usually $70 \%$ ethanol) regardless. The angio-suites are not equipped to work on negative pressure, but only with air filtration system. 


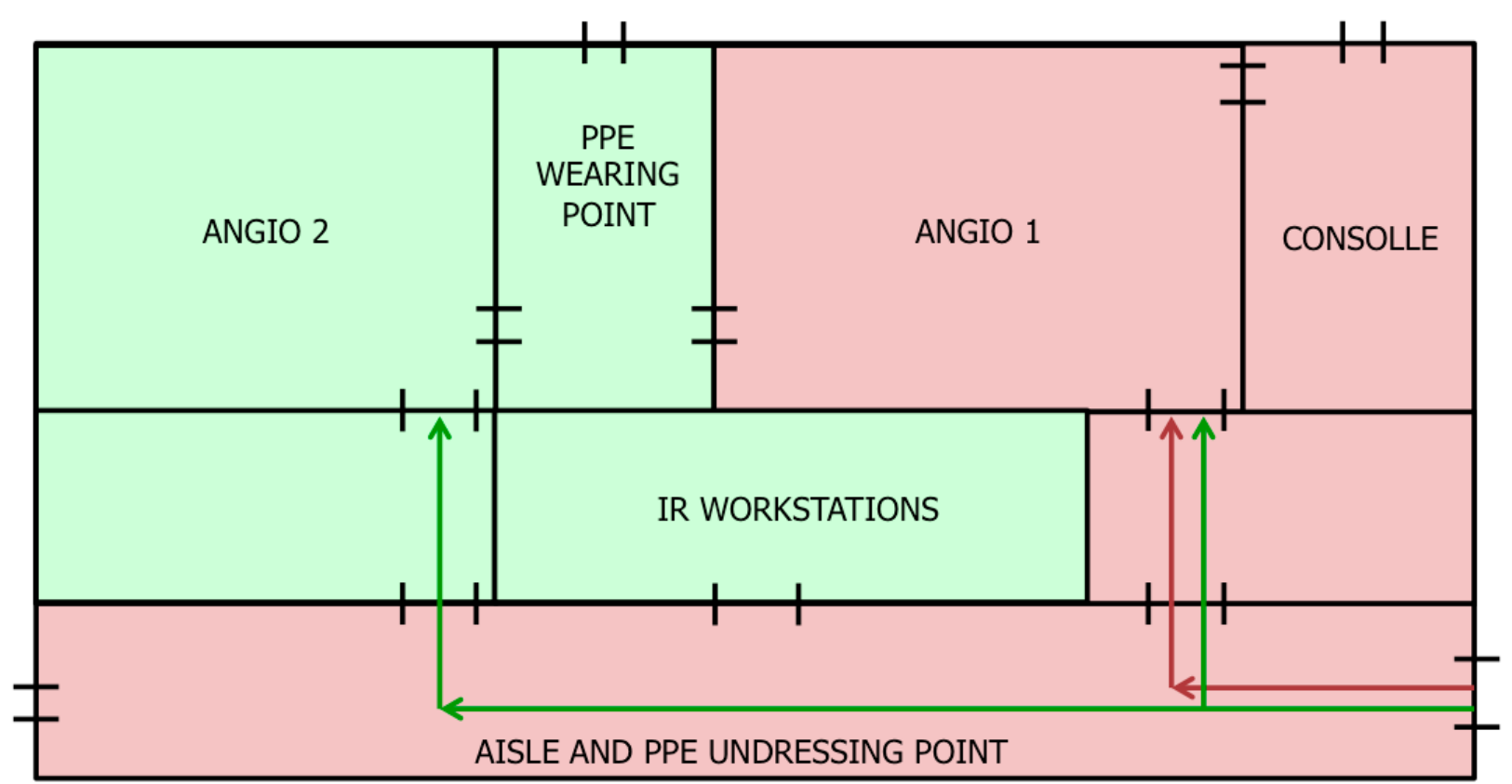

Figure 2. Plan of our IR unit. "ANGIO 1" is the angio-suite with a flat panel digital detector angiograph, while "ANGIO 2" is furnished with a C-arm fluoroscope. The red arrows represent the "COVID-19 route", while the green arrows represent the route of non-COVID-19 patients. Due to the architectural characteristic of our department, it wasn't possible to create a specific route for COVID-19 patients totally separated from the clean one. When a confirmed or suspected COVID-19 patients undergo IR procedures, the red areas can be separeted from the green ones, mantaining them clean. The red areas undergo specific cleaning protocol after each COVID-19 procedure.

\subsection{Population}

Between the $25^{\text {th }}$ of February, when we started to apply the recommended containing measures, and the 14 th of April 2020, when all the IR staff started to undergo nasopharyngeal and oropharyngeal swabs screening, a total of 25 healthcare operators worked at our IR unit.

Operators who, during this period, also worked in other hospital units such as diagnostic emergency department or other healthcare facilities, were excluded from the study.

Applying this criterion, we obtained a final sample of 17 healthcare operators strictly dedicated to IR activity.

None of the operators included in the sample has been in high risk areas, in the previous two weeks, and everyone followed the lockdown rules, imposed by ministerial decrees.

Demographic characteristics and the specific role of each operator are reported in Table 1.

Table 1. Demographic characteristics and job titles of 17 healthcare personnel (HCP) specifically dedicated to IR department in a COVID-Hub Hospital, tested for SARS-CoV-2

\begin{tabular}{cc}
\hline Characteristic & No. (\%) \\
\hline Total HCP & 17 \\
\hline Age in yrs., median (range) & $44.2(26-66)$ \\
\hline Sex & $13(76.5 \%)$ \\
\hline Female & $4(23.5 \%)$ \\
Male & \\
\hline Job title & $5(29.4 \%)$ \\
\hline Interventional radiologist & $1(5.9 \%)$ \\
Dedicated anesthesiologist & $5(29.4 \%)$ \\
Radiology resident & $6(35.3 \%)$ \\
Nurse &
\end{tabular}




\subsection{SARS-CoV-2 Testing}

Nasopharyngeal and oropharyngeal swabs were performed by expert and adequately trained operators to assess the presence of SARS-CoV-2 RNA with real time polymerase chain reaction (RT-PCR) (Charitè, Berlin, Germany) [12]. The same technique was used on both patients and healthcare operators.

After two weeks from the swabs execution, antibodies against SARS-CoV-2 were detected by enzyme-linked immunosorbent assay (ELISA) and chemiluminescence immunoassay (CLIA), based on the recombinant nucleocapsid protein of SARS-CoV-2, on blood samples on IR healthcare operators, whose swabs resulted negative, to evaluate the viral exposition.

\section{Results}

In our Hospital, which has become a COVID-19-Hub, we had a total of 424 confirmed infected SARS-CoV-2 cases between the $25^{\text {th }}$ of February and the 23 of April. Among the confirmed cases, 52 are part of the healthcare staff. Between the 25th of February and the 14th of April 2020, a total of 25 operators worked at our IR unit, but only 17 were specifically dedicated to IR and therefore included in the study. During that period, 12 procedures were performed on patients with confirmed COVID-19 (5 of them were performed bedside) and 11 procedures on patients with suspected COVID-19 (due to the presence of compatible symptoms, compatible CT features or confirmed exposition). Of these cases, 3 patients were then diagnosed as COVID-19 after nasopharyngeal and oropharyngeal swabs, confirmed by SARS-CoV-2 blood tests (specific type of each procedure is reported in Table 2). During all these 23 procedures, every healthcare operator has worn the PPE as specified in previous section.

Table 2. Specific type of each procedure performed on high risk patients between the 25th of February and the 14th of April 2020, sort by date. Two of them (bold) were not initially suspected for COVID-19, but they were diagnosed after the procedure

\begin{tabular}{|c|c|c|c|c|}
\hline Type of procedure & COVID-19 state & High-risk PPE & Bedside & Confirmed COVID-19 \\
\hline Biliary drainage & 2 & $\mathrm{Y}$ & $\mathrm{N}$ & $\mathrm{N}$ \\
\hline Cerebral aneurysm embolization & 2 & $\mathrm{Y}$ & $\mathrm{N}$ & $\mathrm{N}$ \\
\hline Biliary drainage & 3 & $\mathbf{N}$ & $\mathbf{N}$ & $\mathbf{Y}$ \\
\hline Biliary drainage & 2 & Y & $\mathrm{N}$ & $\mathrm{Y}$ \\
\hline Chest drainage & 2 & $\mathrm{Y}$ & Y & $\mathrm{N}$ \\
\hline Infected pancreatic cyst drainage & 1 & $\mathrm{Y}$ & $\mathrm{Y}$ & I \\
\hline $\mathrm{CVC}$ & 2 & $\mathrm{Y}$ & $\mathrm{N}$ & $\mathrm{Y}$ \\
\hline Cholecystostomy & 2 & Y & $\mathrm{N}$ & $\mathrm{Y}$ \\
\hline Infected pancreatic cyst drainage & 1 & $\mathrm{Y}$ & $\mathrm{Y}$ & I \\
\hline Thrombectomy (stroke) & 3 & $\mathbf{N}$ & $\mathbf{N}$ & $\mathbf{Y}$ \\
\hline Hepatic abscess drainage & 1 & $\mathrm{Y}$ & $\mathrm{Y}$ & I \\
\hline Biliary drainage & 2 & $\mathrm{Y}$ & $\mathrm{N}$ & $\mathrm{N}$ \\
\hline Abdominal drainage & 2 & $\mathrm{Y}$ & $\mathrm{N}$ & $\mathrm{N}$ \\
\hline Chest drainage & 1 & $\mathrm{Y}$ & $\mathrm{Y}$ & I \\
\hline Acute lower limb ischaemia & 1 & $\mathrm{Y}$ & $\mathrm{N}$ & I \\
\hline Pulmonary biopsy & 1 & Y & $\mathrm{N}$ & I \\
\hline Symptomatic hydatid hepatic cyst drainage & 2 & $\mathrm{Y}$ & $\mathrm{N}$ & $\mathrm{N}$ \\
\hline Acute lower limb ischaemia & 1 & $\mathrm{Y}$ & $\mathrm{N}$ & I \\
\hline Cholecystostomy & 1 & $\mathrm{Y}$ & $\mathrm{N}$ & I \\
\hline Nephrostomy & 2 & Y & $\mathrm{N}$ & $\mathrm{N}$ \\
\hline Abdominal bleeding embolization & 1 & $\mathrm{Y}$ & $\mathrm{N}$ & I \\
\hline Thrombectomy (stroke) & 2 & $\mathrm{Y}$ & $\mathrm{N}$ & $\mathrm{N}$ \\
\hline Lumbar bleeding embolization & 1 & $\mathrm{Y}$ & $\mathrm{N}$ & I \\
\hline Biliary drainage & 1 & Y & $\mathrm{N}$ & I \\
\hline Abdominal bleeding embolization & 1 & $\mathrm{Y}$ & $\mathrm{N}$ & / \\
\hline
\end{tabular}

Note: COVID-19 state: 1=confirmed (tot. 12); 2=suspected (tot. 11); 3=initially non-suspected (tot. 2).

Confirmed COVID-19: Confirmed or not confirmed suspected COVID-19 patients after the procedure. Yes (Y) or No (N). 
In the same period, 122 procedures were performed on non-suspected COVID-19 patients, all of them with no COVID-19 symptoms and with a first negative swab for SARS-CoV-2 RNA performed at their access to the Hospital. In these cases, operators wore the surgical mask as specified in the internal Hospital guidelines. Out of these patients, 2 developed respiratory symptoms 24-48 hours after the IR procedure and underwent an additional swab which showed the presence of SARS-CoV-2 infection. The 7 dedicated operators involved in those procedures, immediately underwent active surveillance and nasopharyngeal and oropharyngeal swabs. RT-PCR revealed 1 positive and 6 negative result.

In the following days, between the 15th and the 17th of April, all remaining healthcare operators of the IR unit underwent both nasopharyngeal and oropharyngeal swabs, following the screening protocol adopted by the Hospital, with all negative results. The estimated infection rate in our IR unit was 6\%. All operators, underwent SARS-CoV-2 IgG-IgM specific blood test two weeks after the swabs execution that showed all negative results, including the infected one.

Furthermore, we compared the total number of procedures performed at IR unit between the 25th of February and the 14th of April 2020 with the total number of procedures performed between the 25th of February and the 15th of April 2019, considering that 2020 is a leap year. The analysis showed a 33\% decrease of the procedures number $(218$ in 2019 and 145 in 2020), as expected. No differences were found in major/minor procedures ratio between 2019 and 2020 (0.56 in 2019 and 0.5 in 2020).

\section{Discussion}

IR must continue to provide its service even in times of emergency such as during this COVID-19 pandemic and it maintains a fundamental role also in COVID dedicated hospitals.

Our experience shows how COVID-19-Hub Hospital, with a clear reduction of non-COVID patients influx, continued to guarantee numerous urgent and essential procedures, although reducing the workload. By comparing the total number of procedures performed between the 25th of February and the 14th of April 2020 with the total number of procedures performed in the same period of 2019, a clear reduction of IR activities by $33 \%$ was registered (218 in 2019 and 145 in 2020). This significant diminution in the total procedures number was particularly registered once the hospital was converted to a COVID-19-Hub, delegating many procedures to other non-COVID-Hub Hospitals, and strict selecting the most necessary interventions. In the following days, a resumption of the activity was progressively observed along with an increased number of infected patients with comorbidity needing IR procedures.

No differences were found in major/minor procedures ratio between 2019 and 2020 (0.56 in 2019 and 0.5 in 2020), demonstrating that the workload has been globally reduced. A total of 23 procedures (16\%) were performed on patients with suspected or confirmed COVID- 19. In these cases, we applied our internal guidelines, wearing adequate PPE and deeply cleaning and disinfecting rooms and surfaces, as specified in Materials and Methods section, obtaining a prevalence of SARS-CoV-2 infection in our staff members of $6 \%$. This data is encouraging, compared to the $12 \%$ healthcare workers infection prevalence rate reported in Italy [2].

The single case of infected worker was a 35 years old healthy male, interventional radiologist, without any other recognized source of infection. The contagion could possibly be related to the two urgent procedures, both performed by him and other staff members, on patients without suspected COVID-19, during which both patients and operators wore just surgical mask, in addition to the sterile equipment normally used during IR procedures. The first case was a percutaneous trans-hepatic biliary drainage in a patient with sepsis, who underwent two 48-hours spaced nasopharyngeal and oropharyngeal swabs at his hospital access, both negative, and without respiratory symptoms.

The second case, was an already hospitalized patient who underwent endovascular stroke thrombectomy at our IR unit. The patient did not have fever or respiratory symptoms and nasopharyngeal and oropharyngeal swabs, performed at his hospital access, were negative. In both cases, during the following days of hospitalization, patients developed fever, cough, dyspnea and additional nasopharyngeal and oropharyngeal swabs were performed (for the third time in the first case and for the second time in the second case), confirming the presence of SARS-CoV-2 RNA. The infected operator was immediately quarantined and, after two weeks, the infection was considered resolved after two 48-hours spaced negative RT-PCR results. During the isolation period, he was always asymptomatic. He also underwent SARS-CoV-2 IgG-IgM specific blood tests, resulting negative. This result may be related to the test timing, as the IgG-IgM test have been performed just seventeen days after the positive RT-PCR and not thirty days as reported by Long Q et al. [13].

The SARS-CoV-2 IgG-IgM specific blood tests were negative in the remaining 16 operators, suggesting that they weren't effectively exposed to the virus. However, this data has to be taken with caution because the sensibility and specificity of this tests are still under evaluation [14].

These data show that a main issue regarding safety of the staff resides on procedures performed on patients with 
unrecognized COVID 19 diagnosis. At the same time, performing IR procedures on COVID 19 infected patients does not seem to represent a significant increased risk of staff contagion, given that the infection is known and the protection measures are fully applied. Even though, wearing the specific COVID-19 PPE together with lead and sterile equipment is quite uncomfortable for the operators and it might affect their performance especially in long and complex procedures.

Despite functioning in a COVID-19-Hub Hospital, IR units have relatively low exposure to COVID-19 patients, nevertheless to apply preventive measures can further reduce the risk of infection, as demonstrated by our results. This study has some limitation such as the small sample size of the staff included and the overall number of SARS-CoV-2 positive patients treated.

\section{Conclusion}

Our experience demonstrates that applying adequate preventive measures can efficiently reduce the spread of SARS-CoV-2 transmission among the IR staff. The estimated infection in our IR unit was 6\%. Despite a decrease of the IR procedures number is expected in a COVID-19-Hub Hospital, our results point out how the safety measures allow to guarantee the continuity of healthcare service provided by IR, which remain crucial even during the current viral pandemic.

\section{Declaration of Competing Interest}

The authors declare that they have no known competing financial interests or personal relationships that could have appeared to influence the work reported in this paper.

\section{Ethical Approval}

All procedures performed in studies involving human participants were in accordance with the Italian National Research Council (CNR) and with the 1964 Helsinki declaration and its later amendments or comparable ethical standards.

\section{References}

[1] Meselson, M. (2020). Droplets and Aerosols in the Transmission of SARS-CoV-2. New England Journal of Medicine, 382(21), 2063-2063. doi:10.1056/nejmc2009324.

[2] Available online: https://www.epicentro.iss.it/en/coronavirus/sars-cov-2-dashboard (accessed on 1 July 2020).

[3] Odone, A., Delmonte, D., Scognamiglio, T., \& Signorelli, C. (2020). COVID-19 deaths in Lombardy, Italy: data in context. The Lancet Public Health, 5(6), e310. doi:10.1016/s2468-2667(20)30099-2.

[4] Da Zhuang, K., Tan, B. S., Tan, B. H., Too, C. W., \& Tay, K. H. (2020). Old Threat, New Enemy: Is Your Interventional Radiology Service Ready for the Coronavirus Disease 2019? CardioVascular and Interventional Radiology, 43(5), 665-666. doi:10.1007/s00270-020-02440-6.

[5] Ierardi, A. M., Wood, B. J., Gaudino, C., Angileri, S. A., Jones, E. C., Hausegger, K., \& Carrafiello, G. (2020). How to Handle a COVID-19 Patient in the Angiographic Suite. CardioVascular and Interventional Radiology, 43(6), 820-826. doi:10.1007/s00270-020-02476-8.

[6] Chandy, P. E., Nasir, M. U., Srinivasan, S., Klass, D., ... Nicolaou, S. (2020). Interventional radiology and COVID-19: evidence-based measures to limit transmission. Diagnostic and Interventional Radiology, 26(3), $236-240$. doi:10.5152/dir.2020.20166.

[7] Pua, U., \& Wong, D. (2020). What Is Needed to Make Interventional Radiology Ready for COVID-19? Lessons from SARSCoV Epidemic. Korean Journal of Radiology, 21(5), 629. doi:10.3348/kjr.2020.0163.

[8] Mossa-Basha, M., Meltzer, C. C., Kim, D. C., Tuite, M. J., Kolli, K. P., \& Tan, B. S. (2020). Radiology Department Preparedness for COVID-19: Radiology Scientific Expert Review Panel. Radiology, 296(2), E106-E112. doi:10.1148/radiol.2020200988.

[9] Available online: https://www.who.int/emergencies/diseases/novel-coronavirus-2019/situation-reports/ (accessed on 1 July 2020).

[10] Ferioli, M., Cisternino, C., Leo, V., Pisani, L., Palange, P., \& Nava, S. (2020). Protecting healthcare workers from SARSCoV-2 infection: practical indications. European Respiratory Review, 29(155), 200068. doi:10.1183/16000617.0068-2020.

[11] Heinzerling, A., Stuckey, M. J., Scheuer, T., Xu, K., Perkins, K. M., Resseger, H., ... Epson, E. (2020). Transmission of COVID-19 to Health Care Personnel During Exposures to a Hospitalized Patient - Solano County, California, February 2020. MMWR. Morbidity and Mortality Weekly Report, 69(15), 472-476. doi:10.15585/mmwr.mm6915e5. 
[12] Corman, V. M., Landt, O., Kaiser, M., Molenkamp, R., Meijer, A., Chu, D. K., .. Drosten, C. (2020). Detection of 2019 novel coronavirus (2019-nCoV) by real-time RT-PCR. Eurosurveillance, 25(3). doi:10.2807/1560-7917.es.2020.25.3.2000045.

[13] Long, Q.-X., Liu, B.-Z., Deng, H.-J., Wu, G.-C., Deng, K., Chen, Y.-K., ... Cai, X.-F. (2020). Antibody responses to SARSCoV-2 in patients with COVID-19. Nature Medicine, 26(6), 845-848. doi:10.1038/s41591-020-0897-1.

[14] Xiang, F., Wang, X., He, X., Peng, Z., Yang, B., Zhang, J., ... Ma, W.-L. (2020). Antibody Detection and Dynamic Characteristics in Patients With Coronavirus Disease 2019. Clinical Infectious Diseases. doi:10.1093/cid/ciaa461. 